\title{
Étude de différentes méthodes de refroidissement sur le climat et la transpiration de tomates de serre
}

\author{
T Boulard, A Baille, F Le Gall \\ INRA, station de bioclimatologie, BP 91, 84143 Montfavet cedex, France
}

(Reçu le 21 août 1990; accepté le 12 mai 1991)

\begin{abstract}
Résumé - Les effets de différents équipements de climatisation de serre sur la transpiration d'un couvert de tomates et sur le microclimat résultant dans la serre ont été analysés au cours d'expérimentations in situ, où les 3 fonctions de refroidissement suivantes furent étudiées :

- fonction «aération statique»;

- fonction "écran d'ombrage";

- fonction «brumisation».

L'aération statique appliquée seule se traduit, pour les forts renouvellements d'air, par des taux de transpiration élevés liés à l'élévation du déficit de saturation de l'air intérieur. La température de feuillage reste généralement inférieure à la température de l'air, sauf pour les faibles taux de renouvellement.

La fonction ombrage utilisée simultanément avec l'aération statique entraîne des baisses significatives de la transpiration, les températures de feuille et de l'air diminuant légèrement lors de la mise en place de l'écran. L'utilisation simultanée de l'aération et de la brumisation réduit également de façon significative le flux de transpiration, et cela de manière proportionnelle à l'intensité de la brumisation. Le déficit de saturation de l'air de la serre, les températures de l'air et des feuilles subissent également des diminutions significatives.

L'ensemble de ces résultats met en évidence les interactions existant entre les différentes fonctions de climatisation, notamment entre l'aération et la brumisation. lis permettent également d'espérer des améliorations dans la gestion estivale du climat de la serre, à travers la prise en compte en temps réel de la transpiration de la culture.
\end{abstract}

transpiration $/$ Lycopersicon esculentum $=$ tomate $/$ serre $/$ méthode de refroidissement $/$ climatisation $/$ aération

Summary - Study of various cooling methods and their effect on microclimate and transpiration of a greenhouse tomato crop. The effects of various greenhouse cooling devices (fig 1) on greenhouse microclimate and transpiration of a tomato crop (fig 2) have been studied, either separately (static ventilation) or simultaneously (shading screen and ventilation, fog-system and ventilation). The different mechanisms acting on ventilation rate have been analyzed (figs 3, 5):

- buoyancy effect or "stack" effect, due to temperature and humidity differences between inside and outside air;

- wind effect, which induces air exchanges.

Air exchange rates due to these mechanisms have been estimated by means of formulas (eqs 1,2 and 3), and show that the wind effect is predominant (90\% of the total air exchange rate) in most cases. It was found that the shading screen significantly reduced crop transpiration through the decrease in global radiation transmission, while leaf and air temperatures were only slightly reduced when the shading screen was operated (fig 4). Fog system and ventilation, when used simultaneously, led to a significant reduction in crop transpiration, water vapour deficit (fig 5) and air and leaf temperatures (figs 5 and 6). The decrease in crop transpiration was roughly proportional to the intensity of misting.

These experimental results demonstrate the strong interactions between the different cooling devices, mainly between aeration and misting (fig 5). They show that climate management through control of crop transpiration during summer could be significantly improved if these interactions are taken into account in a real-time monitoring system.

transpiration / Lycopersicon esculentum = tomato $/$ greenhouse $/$ coding method $/$ climatisation / static ventilation 


\section{INTRODUCTION}

L'allongement de la période des cultures sous serre a confronté les serristes à des problèmes de climatisation inhérents aux forts contrastes de température et d'hygrométrie régnant sous serre en période estivale, notamment dans les zones du pourtour méditerranéen, à climat chaud et sec. L'utilisation de techniques de climatisation telles que l'aération statique (ouvrants) ou dynamique (extracteurs d'air), les écrans d'ombrage ou le refroidissement évaporatif (panneaux évaporants ou "cooling", brumisation, etc) est actuellement courante chez les serristes. Cependant, leur gestion est encore mal maîtrisée dans la plupart des exploitations, du fait du manque de connaissances sur les modifications des flux intérieurs de chaleur et de masse $\left(\mathrm{H}_{2} \mathrm{O}, \mathrm{CO}_{2}\right.$, etc) entraînées par l'utilisation, souvent simultanée, de ces équipements. Ainsi, les interactions aération/ombrage ou aération/brumisation sont mal connues et ne permettent pas d'inclure, dans les systèmes de gestion automatisée, des algorithmes qui les prennent en compte.

Les données disponibles sur les effets de ces techniques de climatisation sont actuellement limitées aux variables d'état (température, hygrométrie) ou aux flux radiatifs solaires (Brun et Lagier, 1984; Baille et al, 1985). Très peu de données sont disponibles sur les flux de chaleur sensible et latent, et sur leur importance respective dans le refroidissement ou le réchauffement de la serre.

Les objectifs de l'étude présentée visent donc à mieux appréhender les mécanismes d'action de ces techniques de refroidissement sur le comportement thermodynamique du système serre-culture, et notamment sur les flux de chaleur latente. En effet, la transpiration du couvert et l'évaporation du sol ont un rôle prépondérant dans les mécanismes de refroidissement de la serre : l'énergie excédentaire ou "charge solaire" à évacuer en été sous une serre représente des flux de l'ordre de $500-600 \mathrm{~W} / \mathrm{m}^{2}$ aux heures les plus chaudes. L'évapotranspiration de la culture, de l'ordre de $60-70 \%$ de ce flux lorsque la culture transpire librement (Boulard, 1985), joue donc le rôle principal dans la transformation et la dissipation de l'énergie solaire excédentaire. La stratégie la plus naturelle pour refroidir la serre consiste donc à maximiser la transpiration naturelle de la culture (irrigation optimale, système racinaire développé, substrat adéquat, etc), avec l'avantage supplémentaire de maintenir la plante dans des conditions physiologiques optimales. Par contre, toute action modifiant cette transpiration à la baisse, sans influer significativement sur la charge solaire, aura des conséquences négatives sur le climat interne, et risquera d'entraîner des stress d'origine thermique ou hydrique au niveau de la culture.

Les 3 "fonctions" de refroidissement abordées dans cette étude sont :

- la fonction «aération statique»;

- la fonction «écran d'ombrage»;

- la fonction «brumisation».

L'approche expérimentale choisie a été la suivante : l'aération étant toujours utilisée en combinaison avec l'une des 2 autres fonctions, nous avons d'abord étudié l'influence de la seule fonction "aération». Dans les étapes suivantes, le niveau d'aération a été fixé à une valeur constante et les actions combinées aération/écran d'ombrage et aération/brumisation ont été réalisées sans chercher à respecter des consignes strictes de température et d'hygrométrie, mais en maintenant le climat intérieur dans des normes acceptables de confort pour les cultures.

\section{MATÉRIEL ET MÉTHODES}

\section{Dispositif expérimental}

\section{La serre et la culture}

Les expérimentations ont été conduites en période estivale (fin mai-fin août 1989) dans une serre bichapelle de $416 \mathrm{~m}^{2}$ à couverture de polyéthylène (fig 1), située sur le domaine expérimental du centre de recherches d'Avignon $\left(44^{\circ} \mathrm{N}\right)$.

Cette serre est équipée :

- de 2 ouvrants en toiture sur toute la longueur de la serre (fig 1);

Ce dispositif est représentatif des dispositifs d'aération existant dans les serres commerciales. Si $\alpha$ représente l'angle d'ouverture, I la largeur de l'ouvrant et $L$ sa longueur, on déduit les paramètres suivants :

- $H(\mathrm{~m})$ la hauteur de l'ouverture : $H=I . \sin \alpha$

- $S_{0}\left(m^{2}\right)$ la surface de l'ouverture : $S_{0}=$ L.I. $\sin \alpha$

- $r(\%)$ le pourcentage du surface ouverte par rapport à la surface au sol $S$ de la serre :

$$
r_{s}=2 S_{0} / S .100
$$

(le coefficient 2 est pris pour 2 ouvrants).

Pour notre serre expérimentale, nous avons : $l=$ $1,05 \mathrm{~m}, L=32 \mathrm{~m}, S=416 \mathrm{~m}^{2}$. Par la suite, nous utiliserons systématiquement le paramètre $r$ pour traduire le degré d'ouverture de la serre;

- d'un écran d'ombrage partiellement aluminisé (type LS 15; constructeur Ludwig et Svenson), possédant une transmittance d'environ $40 \%$ et une réflectance de 
$50 \%$ au rayonnement global solaire, tendu horizontalement à hauteur des cheneaux $(3 \mathrm{~m})$ et ménageant un passage de $1 \mathrm{~m}$ de large au centre de chaque chapelle (fig 1);

- d'un système de brumisation (fig 1) à haute pression (40 bars), de type "fog-system" (Société TII Orange 84), émettant à partir de buses très fines des gouttelettes d'eau dont le spectre s'étend de $10 \mu \mathrm{m}$ à $50 \mu \mathrm{m}$ (données du constructeur). La densité des buses, situées sur des rampes d'alimentation juste au-dessous de l'écran d'ombrage, est de 1 buse pour $6,4 \mathrm{~m}^{2}$ de sol, avec un débit maximal de $7,2 \mathrm{l} / \mathrm{h}$ et par buse, soit $1,11 \cdot h^{-1} \cdot m^{-2}$.

La gestion de l'ensemble de ces équipements est assurée par un ordinateur de climatisation (société Edenland, Manosque 04), où les consignes de température et d'hygrométrie sont implantées et modifiées selon les besoins de l'expérimentation. Des capteurs de position des ouvrants appartenant à cette chaîne de climatisation permettaient de connaître la position des ouvrants.

La culture pratiquée était une culture de tomates (cv Duranto) plantée en janvier, à une densité d'environ 2,5 plantes $/ \mathrm{m}^{2}$, et palissée sur cordon. Au moment des essais (mi-juin), le couvert se présentait sous forme de rangées de $2 \mathrm{~m}$ de haut, de $0,9 \mathrm{~m}$ de large et séparées par les allées de $1,1 \mathrm{~m}$. Le LAI $\left(\mathrm{m}^{2}\right.$ de feuilles $/ \mathrm{m}^{2}$ de sol) mesuré à cette époque était de 1,9.

\section{Les mesures}

\section{Mesures climatiques}

Le plan du dispositif expérimental de mesures climatique et de transpiration est présenté sur la figure 2. Les mesures de température et d'hygrométrie sont réali- sées à l'aide de psychromètres ventilés situés à différentes hauteurs dans la serre. Les rayonnements solaires globaux à l'intérieur et à l'extérieur (piles Kipp et Zonen), la vitesse et la direction du vent sont enregistrés sur une acquisition de données portable (Campbell $\times 21$ ), qui réalise une moyenne sur une période de $5 \mathrm{~min}$. Ces données moyennes sont stockées, puis transférées sur microordinateur pour le traitement ultérieur.

\section{Les températures de feuillage}

Elles ont été acquises à l'aide de thermocouples extrêmement fins, reliés en série à chaque étage du couvert et piqués sur la face inférieure des feuilles au niveau de la nervure principale du foliole terminal. Ces mesures ont été opérées à 3 niveaux du couvert $(0,5 \mathrm{~m}, 1,2 \mathrm{~m}, 1,9 \mathrm{~m})$, à proximité (moins de $1 \mathrm{~m}) \mathrm{du}$ lysimètre. Une valeur moyenne de référence de température de feuille a ensuite été calculée à partir de ces 3 mesures élémentaires.

\section{Mesure de la transpiration}

Elle est réalisée au moyen d'un lysimètre implanté dans la continuité des rangs de culture (figs 1 et 2), et supportant 4 plants de tomate. Le lysimètre est constitué d'une balance électronique (marque Sauter, précision de $1 \mathrm{~g}$ pour une portée de $120 \mathrm{~kg}$ ) sur laquelle est disposé un chassis supportant le substrat et un portique assurant le tuteurage des plants. Deux réservoirs (pour le drainage et l'irrigation) sont également solidaires du chassis. Si on néglige l'évaporation du substrat, qui est très faible du fait du recouvrement des bacs de culture par un film plastique, et l'accroissement en poids sec des plantes, la diminution du poids de l'ensemble ainsi formé correspond à la transpiration des plantes de tomate portées par le chassis.

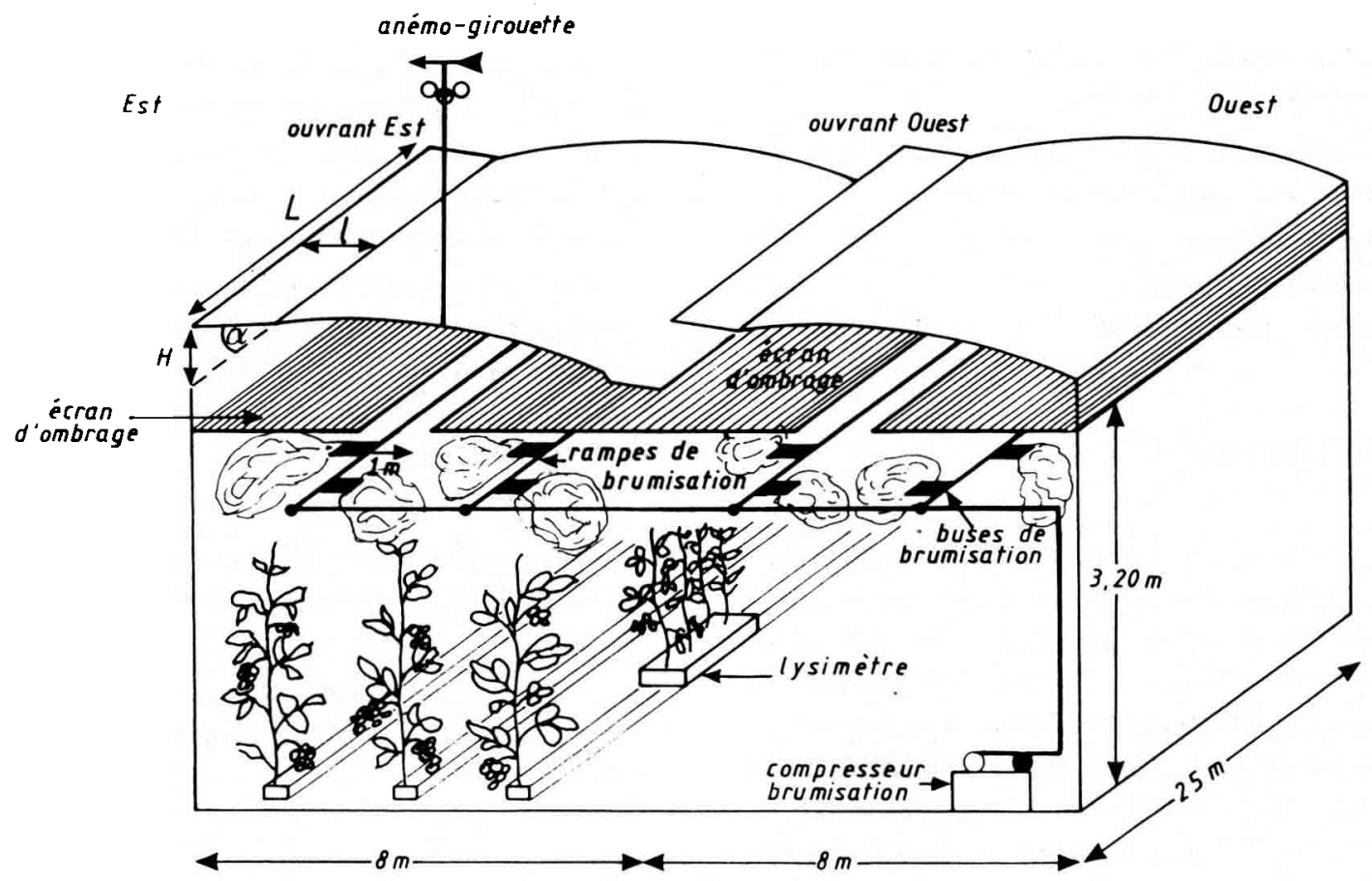

Fig 1. Schéma de la serre expérimentale $\left(416 \mathrm{~m}^{2}\right)$ et des dispositifs de climatisation testés. Caractéristiques des ouvrants: $\alpha=$ angle d'ouverture, $I=1,05 \mathrm{~m}, L=32 \mathrm{~m}$. 


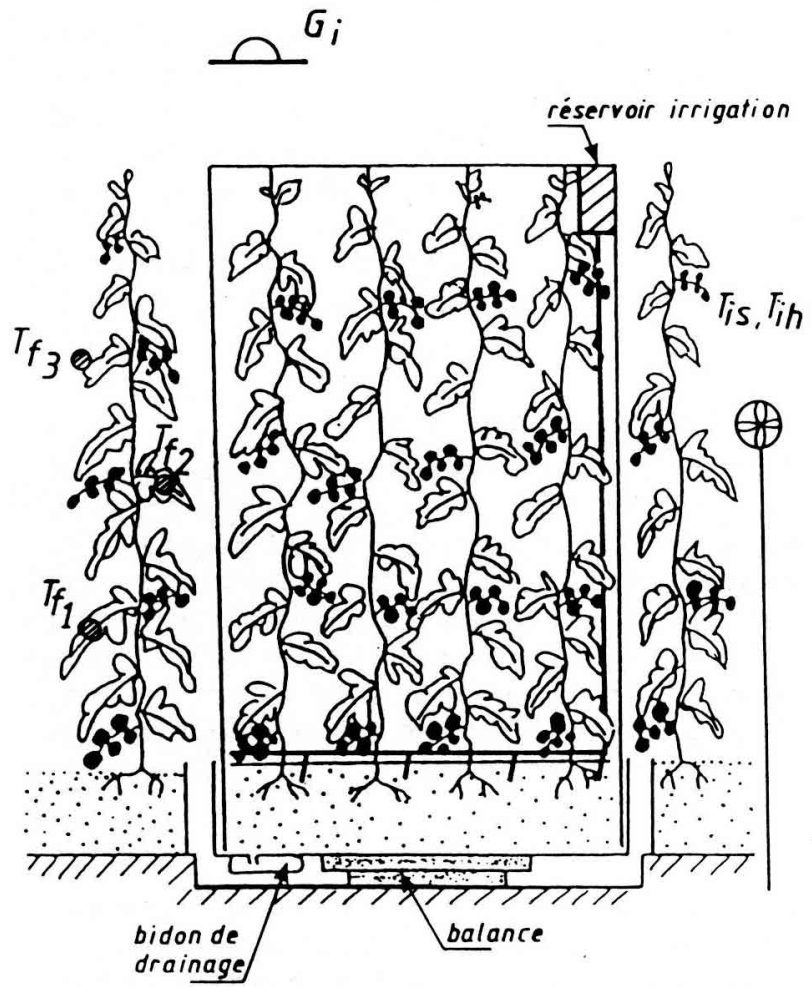

Fig 2. Schéma des installations de mesures lysimétriques et climatiques installées sous les serres. $G_{i}$ : pile de rayonnement global située au-dessus de la culture; $T_{i s}, T_{i h}$ : psychromètre; $T_{f 1}, T_{f 2}, T_{f 3}$ : mesures de températures de feuille situées à 3 étages du couvert $(0,5 \mathrm{~m}, 1,2 \mathrm{~m}, 1,9 \mathrm{~m})$.

\section{RÉSULTATS}

Pendant la période juin-juillet 1989 , un certain nombre de journées-test, au cours desquelles nous avons étudié les différentes variantes, ont été analysées dans le détail.

Nous présenterons ci-dessous les résultats concernant les 3 variantes suivantes:

a : aération seule;

$b$ : aération + ombrage;

$c$ : aération + brumisation.

\section{Journée «aération»}

Lors de cette journée ensoleillée ( $20^{\circ}$ juin 1989), des séquences successives d'une demi-heure de durée d'ouverture, à des degrés variables, des ouvrants situés en toiture ont permis de mettre en évidence les effets du taux de renouvellement d'air de la serre sur la transpiration de la culture, TR, $\left(\mathrm{W} / \mathrm{m}^{2}\right)$ : la figure $3 a$ présente l'évolution de TR, $G_{0}$, rayonnement global extérieur, et $G_{i}$, rayonnement global incident sur la culture au cours de cette journée.
$\grave{A}$ ouverture maximale $(r=10 \%)$, le niveau de transpiration est maximal, de l'ordre de 250-300 $\mathrm{W} / \mathrm{m}^{2}$, ce qui représente environ $45 \%$ du rayonnement incident, $G_{j}$. Les faibles ouvertures $(r<$ $2 \%)$ entraînent une diminution très nette de TR, de l'ordre de $50-60 \%\left(T R \approx 100 \mathrm{~W} / \mathrm{m}^{2}\right)$. Cette diminution semble fortement corrélée avec la diminution du déficit de saturation intérieur, $D_{i}$, qui passe de $12 \mathrm{mbar}$ pour $r=10 \%$, à environ 2 mbar pour $r=1 \%$ (fig $3 b$ ), où l'on est donc très proche de la saturation, alors que le déficit extérieur $D_{0}$ reste constant, de l'ordre de 15 mbar. Les diminutions de $D_{i}$ avec les ouvertures intermédiaires $(r=2,7$ et $5,3 \%)$ sont également significatives.

Si l'on analyse les effets de l'aération sur les températures d'air sous serre, $T_{i}$, et du feuillage, $T_{f}$, on observe (fig $3 \mathrm{c}$ ) que la température $T_{f}$ est généralement inférieure à la température de l'air, notamment pendant les périodes de grande ouverture $(r>5 \%)$, mais lui devient supérieure lors des périodes de faible ouverture $(r<2 \%)$. Cette inversion de la différence $\left(T_{f}-T_{i}\right)$ montre donc une plus grande sensibilité de la température de feuille aux variations du taux de renouvellement de la serre. Ces augmentations de $T_{f}$ correspondent en fait aux diminutions de la transpiration $T R$ et du déficit de saturation $D_{i}$ observés lors du passage aux faibles angles d'ouverture. On peut aussi remarquer, pour des ouvertures d'ouvrants identiques, que la température d'air $T_{i}$ est proche de la température extérieure $T_{0}$ lorsque la vitesse du vent est faible (en matinée), mais est inférieure de $1^{\circ} \mathrm{C}$ environ dans l'après-midi lorsque le vent est plus fort (accroissement de 2 à 5 $\mathrm{m} . \mathrm{s}^{-1}$ ). Le pourcentage d'ouverture ne suffit donc pas à expliquer tous les phénomènes observés et la détermination exacte du renouvellement d'air de la serre est indispensable.

Le flux volumique d'air $\left(G_{v}\right)$ pénétrant ou sortant d'une serre équipée d'ouvrants situés en position haute peut être calculé en prenant en compte les 2 forces motrices principales de l'aération statique :

- l'effet de la pression du vent extérieur;

- l'effet «cheminée» dû à la différence de température, donc de densité, entre l'air extérieur et intérieur.

Pour le type utilisé, avec 2 ouvrants en toiture (fig 1), Kabbaj (1988) propose la relation suivante :

$$
F_{V}=A I . \frac{S}{2} \cdot\left(2 \cdot g \cdot \frac{|D T|}{T_{i}} \cdot \frac{H}{4}+C \cdot V^{2}\right)^{0,5}
$$




\section{(o)}

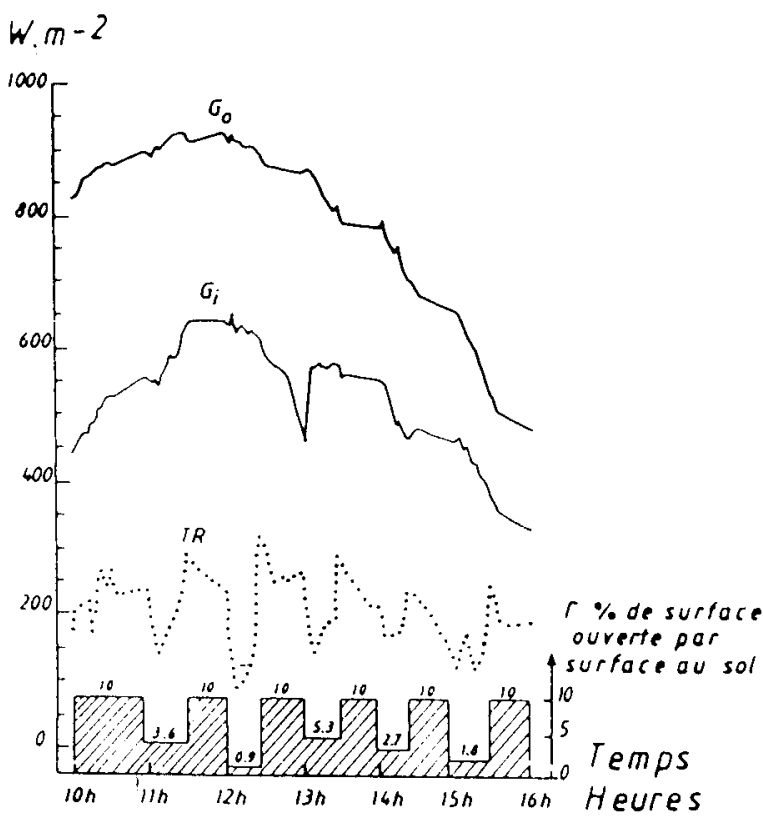

(c)

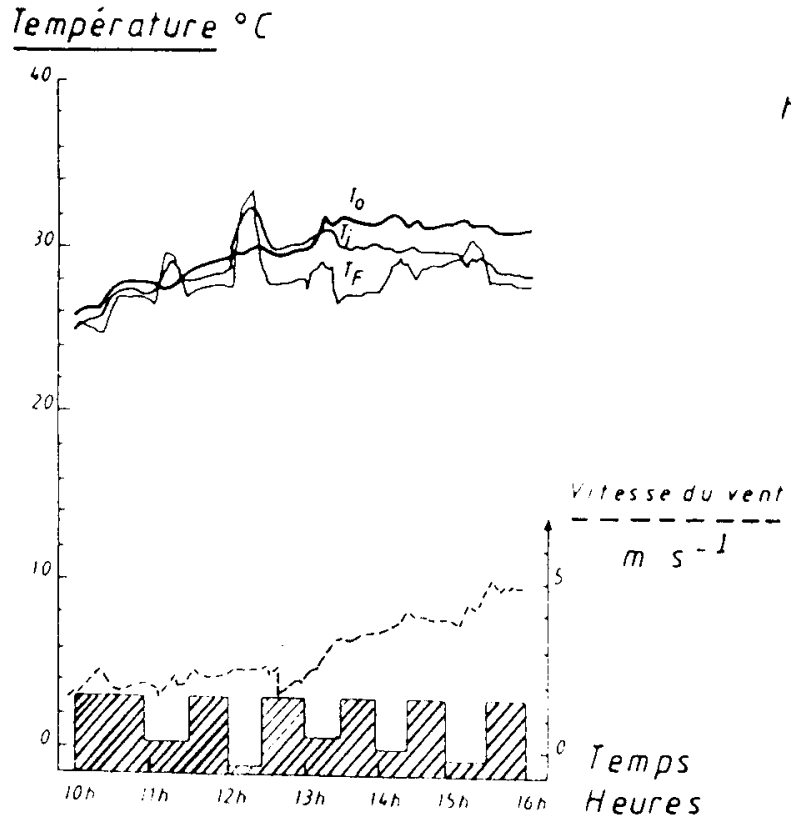

(b)

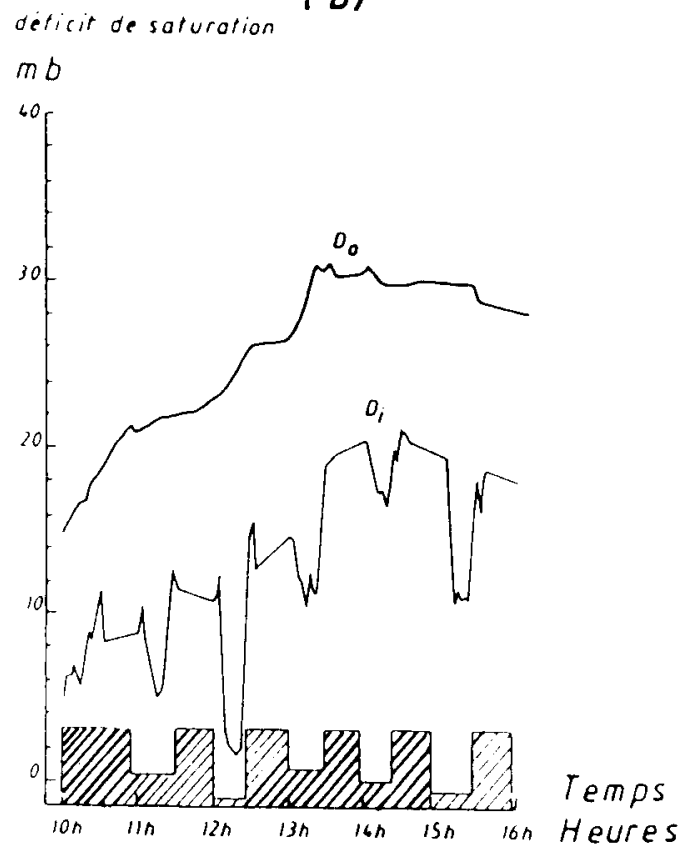

(d)

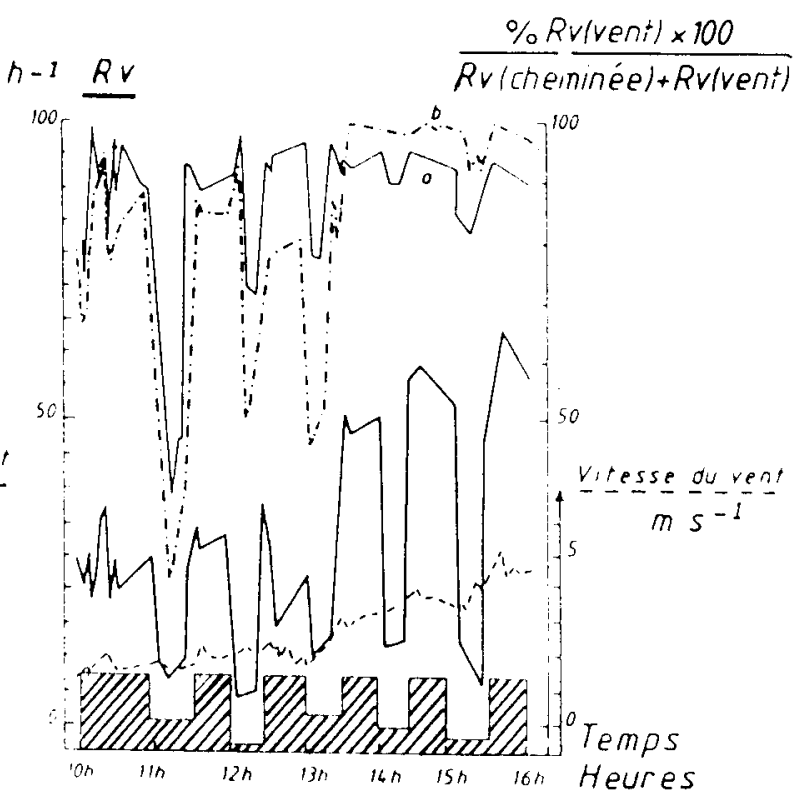

Fig 3. Étude de l'aération statique. Journée du 20 juin 1989 à Montfavet. Effet de différentes ouvertures des ouvrants $r$ (exprimées en $\%$ de surface ouverte par surface de sol) : a) sur la transpiration de la culture TR. $G_{0}$ : rayonnement global extérieur; $G_{i}:$ rayonnement global sous serre. b) sur le déficit de saturation de l'air de la serre $D_{i} . D_{0}$ : déficit de saturation de l'air extérieur. c) sur la température d'air de la serre, $T_{j}$; et des feuilles, Tf. La vitesse du vent extérieur $V$ et la température extérieure $T_{0}$ sont également reportées sur le graphique. d) évolution du renouvellement d'air calculé $R_{v}$, et de sa composante advective, $R_{v}\left(\right.$ vent) $/\left(R_{v}\right.$ (cheminée) + $R_{v}\left(\right.$ vent)), $R_{v}$ (cheminée) étant calculé soit à partir de $\mid D T / / T\left(\right.$ a) soit à partir de $\mid D T_{v} / / T_{v}$ (b)

avec:

$F_{v}$ : flux volumique d'air $\left(\mathrm{m}^{3} \cdot \mathrm{s}^{-1}\right)$;

$S$ : surface ouverte $\left(\mathrm{m}^{2}\right)$;

$g$ : accélération de la pesanteur $\left(\mathrm{m} . \mathrm{s}^{-2}\right)$;

$D T$ : différence de température intérieurextérieur:
$H$ : hauteur de la surface ouverte des ouvrants $(\mathrm{m})$;

$T_{i}$ : température intérieure $\left({ }^{\circ} \mathrm{K}\right)$;

$V$ : vitesse du vent $\left(\mathrm{m}_{\mathrm{s}} \mathrm{s}^{-1}\right)$;

$A l$ : coefficient de perte de charge des ouvrants;

$C$ : coefficient de pression du vent. 
Le renouvellement d'air de la serre $R_{v}$ se déduit de $F_{v}$ par la relation:

$$
R_{V}=\frac{F_{v} \cdot 3600}{\text { Volume de la serre }}\left(\mathrm{h}^{-1}\right)
$$

Dans la relation (1), le premier terme de la somme entre parenthèses représente la contribution de l'effet "cheminée" au renouvellement de l'air de la serre, alors que le second (C.V2) traduit la contribution de l'effet du vent extérieur.

Pour la serre qui sert de support à cette étude, les coefficients ont été identifiés in situ à partir de mesures à l'aide d'un gaz traçeur, $\mathrm{N}_{2} \mathrm{O}$ (Kabbaj, 1988).

Des valeurs de renouvellement de l'air de la serre ont été calculées (fig 3c,d) au cours de la journée "aération»; elles varient de $10 \mathrm{~h}^{-1}$ (faibles ouverture et vent $\approx 3 \mathrm{~m} \cdot \mathrm{s}^{-1}$ ) à plus de $60 \mathrm{~h}^{-1}\left(r=10 \%\right.$, vent de 5 m.s. $\left.{ }^{-1}\right)$.

On peut observer que, pour une même ouverture $(r=10 \%), R v$ passe de $25 \mathrm{~h}^{-1}$ avec un vent de $2,5 \mathrm{~m} . \mathrm{s}^{-1}$ à plus de $50 \mathrm{~h}^{-1}$ pour un vent de 5 m.s. ${ }^{-1}$ en fin d'après-midi. On peut également mesurer la contribution du vent au renouvellement de l'air de la serre par l'intermédiaire du rapport :

$$
C V /\left[C V^{2}+2 g \cdot \frac{|D T|}{T_{i}} \cdot \frac{H}{4}\right]
$$

On remarque (fig $3 d$ ) que cette contribution est largement prépondérante ( $90 \%$ du total) tant que le vent est fort et l'angle d'ouverture des ouvrants grand; inversement, pour des vents faibles et des ouvertures réduites, la valeur de $D T$ augmente, accroît l'effet cheminée et contribue à freiner l'augmentation de la température de la serre.

Dans ce qui précède, l'effet cheminée est traduit uniquement par la différence de température entre l'intérieur et l'extérieur. Or, les modifications d'ouverture des ouvrants s'accompagnent également d'une variation importante de l'écart

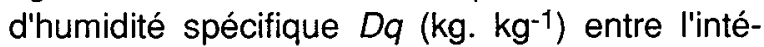
rieur et l'extérieur. Cet écart doit être pris en compte, l'air humide étant plus léger que l'air sec. Pour cela, on utilisera l'écart de température virtuelle, $T_{v}$ (Souloumiac et Itier, 1989) avec :

$$
T_{v}=T(1+0,61 q)
$$

$$
\text { On substituera donc } \mid D T / \text { par }\left|D T_{v}\right|
$$

$$
\left|D T_{v}\right|=\left|D T+\left(\left(T_{i}+T_{o}\right) / 2\right) \cdot 0,61 D q\right|
$$

Si on examine l'effet de $D q$ sur le renouvellement d'air (figure 3d), on constate que :

- si $T_{i}$ est proche de ou plus grand que $T_{0}$ (vent faible, faibles ouvertures d'ouvrants), une augmentation de l'écart $D q$ se traduit par une augmentation de $\left|D T_{v}\right|$, et donc de la contribution de l'effet cheminée;

- par contre si $T_{i}$ est inférieur à $T_{o}$ (vent fort) les signes de $D T$ et $D q$ vont être de sens opposé et $\mid D T_{v} /$ sera plus faible que $|D T|$. L'écart de densité due à la différence d'humidité spécifique va s'opposer à l'effet cheminée.

\section{Journée «ombrage»}

Des séquences successives (durée d'environ $1 \mathrm{~h}$ ) de déploiement et de repliement de l'écran d'ombrage ont été effectuées lors d'une autre belle journée ensoleillée, peu ventée (journée du 29 mai 1989), l'ouverture des ouvrants restant fixée à une valeur de $r=5,3 \%$ pendant tout la journée. Les résultats obtenus pour $T R, G$ et $G_{i}$ sont présentés sur la figure $4, a$ : on constate que la mise en place de l'écran s'accompagne d'une nette diminution de la transpiration, de l'ordre de 40$50 \%$, inférieure cependant à la réduction du rayonnement global, qui est de l'ordre de $60 \%$.

Lors de la deuxiěme période de déploiement de l'écran vers $12 \mathrm{~h}$, on assiste à une forte chute du déficit de saturation de l'air intérieur, $D_{i}$, de 12 mbar à 1 mbar (fig $4 b$ ), entraînant une situation proche de la saturation.

Cette situation est sans doute due à un taux de renouvellement très faible de la serre, du fait de la vitesse de vent pratiquement nulle. II ne nous a pas été possible de vérifier cette hypothèse en recalculant le taux de renouvellement de l'air de la serre à l'aide de la relation (1), les coefficients $A$ l et $C$ ayant été identifiés en l'absence d'écran d'ombrage. Les baisses de déficit de saturation sont moins importantes lors des autres périodes de déploiement de l'écran, notamment dans l'après-midi où la vitesse du vent, plus élevée, permet des échanges plus intenses entre l'intérieur de la serre et l'extérieur, maintenant ainsi $D_{i}$ à des niveaux plus élevés.

La température de feuillage (fig 4c) au cours de cette journée, reste inférieure à la température d'air, de l'ordre de $2^{\circ}-3^{\circ} \mathrm{C}$. Le déploiement 


\section{(a)}

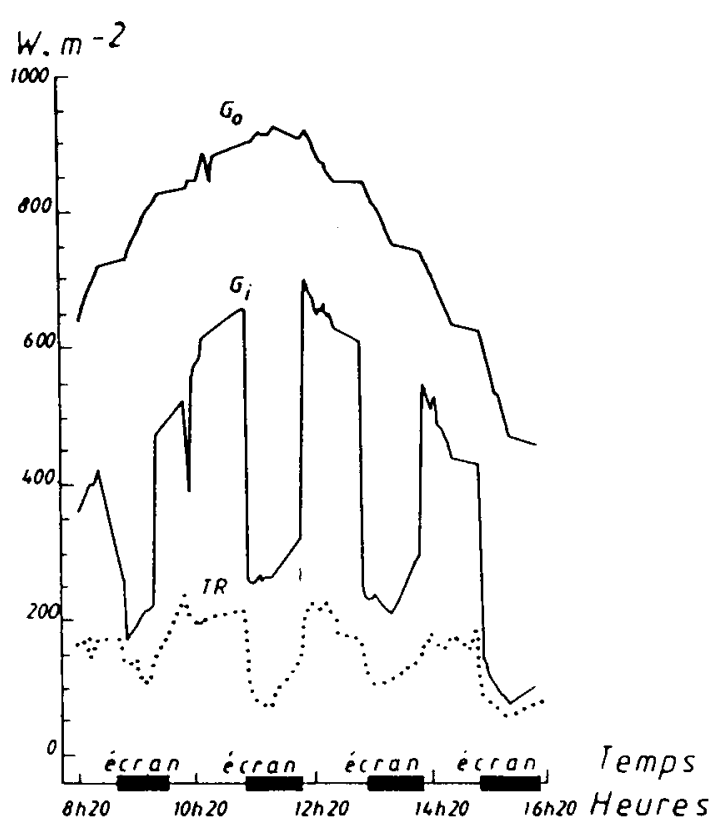

(b)

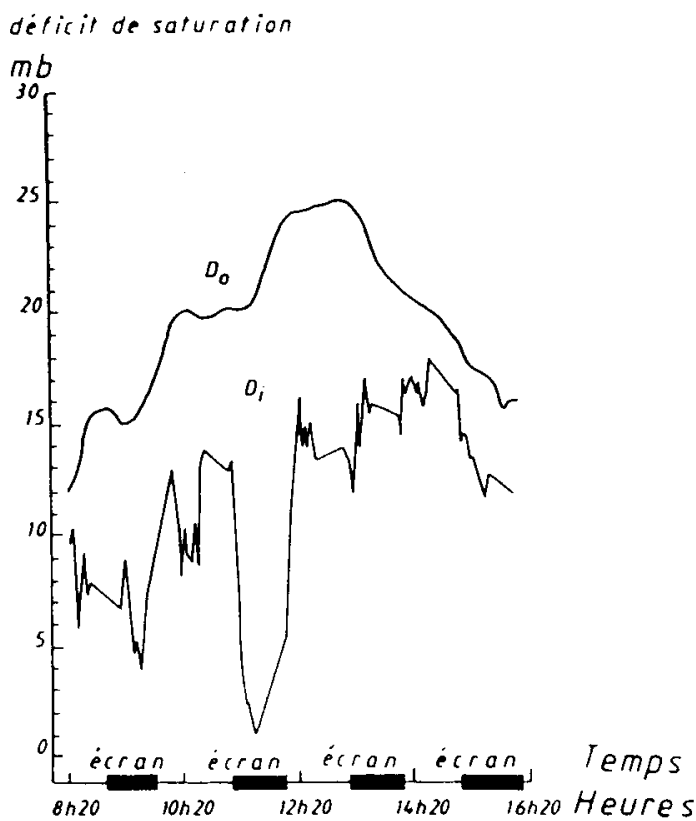

(c)

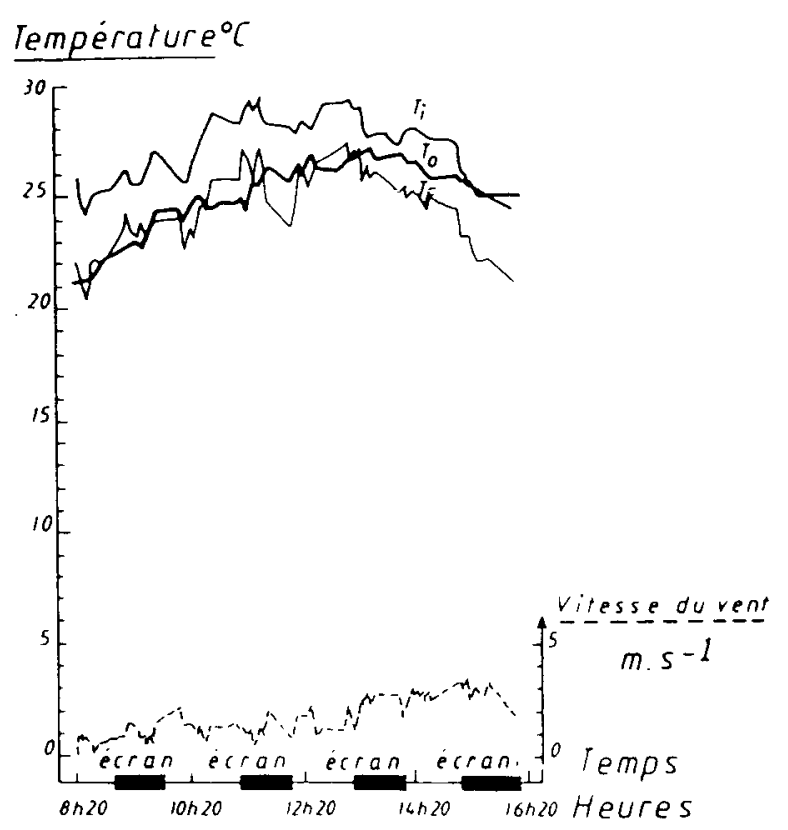

Fig 4. Étude de l'effet de l'ombrage. Journée du 29 mai 1989 à Montfavet $(r=5,3 \%)$. a) sur le rayonnement sous serre $G_{j}$, et sur la transpiration de la tomate : TR; $G_{0}$ : rayonnement global extérieur. b) sur le déficit de saturation de l'air de la serre $D_{i} ; D_{0}:$ déficit de saturation de l'air extérieur. c) sur la température d'air de la serre $T_{i}$ et de feuille $T_{f}$; la vitesse du vent extérieur $V$ et la température extérieure $T_{0}$ sont également reportées sur le graphique.

de l'écran entraîne une baisse des températures d'air et de feuillage de l'ordre de $1-2{ }^{\circ} \mathrm{C}$ (sauf pour la 1re période de déploiement de l'écran, où l'effet de l'ombrage n'est pas net, du fait sans doute de la montée rapide en température de la serre en cette période matinale).
Par rapport à la température extérieure $T_{0}$, la température intérieure $T_{i}$ montre un écart positif le matin, qui s'annule vers $15 \mathrm{~h} 00$. La température de feuillage $T_{f}$ reste proche de la température $T_{0}$ jusqu'aux environs de 13 h00 (moment du $3^{e}$ déploiement), puis diminue beaucoup plus 
rapidement que $T_{0}$ et $T_{j}$, pour atteindre un écart de $-4{ }^{\circ} \mathrm{C}$ par rapport à $T_{o}$ à la fin de la période du 4 e déploiement.

\section{Journée «brumisation»}

La journée choisie (31 mai 1989) est une journée ensoleillée jusque vers $13 \mathrm{~h}$, très couverte ensuite (fig 5a). Des périodes successives de brumisation ont été programmées toutes les h, avec fonctionnement pendant $30 \mathrm{~min}$. Les intensités de brumisation (en $s$ de fonctionnement pour 1 min d'arrêt) ont été successivement de 10 s, $30 \mathrm{~s}, 1 \mathrm{~min}, 40 \mathrm{~s}, 20 \mathrm{~s}$ et enfin $2 \mathrm{~min}$, qui est l'intensité maximale, appliquée un peu avant l'arrivée de la couverture nuageuse. Les ouvrants étaient maintenus à une ouverture fixe $(r=$ $5,3 \%)$.

La vitesse du vent s'établissant à une valeur constante (entre 4 à $5 \mathrm{~m}^{-\mathrm{s}^{-1}}$ ) à partir de $10 \mathrm{~h}$, le taux de renouvellement de l'air de la serre (fig $5 \mathrm{~d})$ demeure également constant $\left(\approx 25 \mathrm{~h}^{-1}\right)$. Les seules variations perceptibles concernent la contribution de l'effet de cheminée au renouvellement d'air total de la serre, celui-ci diminuant et s'annulant presque lors de la séquence de brumisation la plus forte à la suite de l'inversion de l'écart de $D T$ entre intérieur et extérieur (fig $5 c$ ) qui prend un signe opposé à celui de $D q$ et entraîne la diminution de $\left|D T_{v}\right|$.

Les diminutions de transpiration observées lors des périodes de brumisation (fig $5 \mathrm{a}$ ) semblent grosso-modo proportionnelles aux intensités du brumisation. Avec les intensités les plus élevées ( $1 \mathrm{~min}$ et $40 \mathrm{~s}$ ), correspondant respectivement à des apports de 0,55 et $0,32 \mathrm{~mm} / \mathrm{h}$, on observe une diminution d'environ $30 \%$ de TR, qui passe de $300 \mathrm{~W} / \mathrm{m}^{2}$ à $200 \mathrm{~W} / \mathrm{m}^{2}$ (c à $\mathrm{d}$ de $0,44 \mathrm{~mm} / \mathrm{h}$ à $0,29 \mathrm{~mm} / \mathrm{h}$ ).

On observe simultanément une baisse du déficit de saturation $D_{i}$ (fig $5 \mathrm{~b}$ ), qui ramène le déficit à des valeurs de l'ordre de 5 à 7 mbar alors qu'il peut atteindre 20 mbar lors des périodes sans brumisation.

La température de l'air dans la serre diminue également de manière significative, de l'ordre de $3-4{ }^{\circ} \mathrm{C}$ pour les intensités de brumisation les plus élevées (fig $5 \mathrm{c}$ ).

Les mesures de température de feuilles ayant été défaillantes pour la journée du 31 mai 1989 nous avons analysé l'évolution de ce paramètre à partir des courbes d'une autre journée «brumisation», celle du 21 juin 1989 (fig 6a). On peut observer que la température de feuille suit assez fidèlement les évolutions de la température d'air avec $3-4{ }^{\circ} \mathrm{C}$ de moins, quelle que soit l'intensité de brumisation. On constate cependant une exception lorsque le renouvellement d'air est faible (vent faible) et que l'on impose simultanément une durée de brumisation importante (première séquence de brumisation de 60 $\min )$ : dans ce cas, le déficit de saturation chute (fig 6 b) et la température de feuille s'établit au même niveau que celle de l'air de la serre.

\section{DISCUSSION}

Les premières conclusions qu'il est possible de retirer de ces résultats expérimentaux réalisés sous serre avec des cultures de tomates bien alimentées en eau sont les suivantes:

\section{L'aération est utilisée seule}

Le taux de renouvellement de la serre $R_{v}$ influe de manière très nette sur le taux de transpiration de la culture. Pour des faibles valeurs de renouvellement (vent nul ou faible, petits angles d'ouverture), les échanges avec l'extérieur sont très ralentis: I'hygrométrie de la serre augmente rapidement du fait de la transpiration de la culture, induisant alors par l'effet "feedback", une diminution de la transpiration (action sur la composante advective de la transpiration, la composante radiative n'étant pas affectée) et une augmentation de la température du feuillage, qui peut alors être supérieure à celle de l'air. Aux valeurs plus fortes de renouvellement (vent fort, grand angle d'ouverture), les échanges avec l'extérieur permettent d'évacuer suffisamment d'énergie sensible et latente pour maintenir des valeurs de $D_{i}$ élevées et, donc, des taux de transpiration qui peuvent être 2 fois plus élevés que ceux obtenus avec les faibles renouvellements d'air : la température de feuillage redevient alors inférieure à la température d'air.

\section{Aération et ombrage}

La mise en place d'un écran d'ombrage entraîne des baisses significatives (diminution de moitié) de $T R$, dont les 2 composantes, advective et radiative, sont cette fois-ci affectées de façon plus ou moins importante. Lorsque le taux de renouvellement est suffisamment élevé (vent fort), l'effet de l'écran sur $D_{i}$ n'est pas très net (diminu- 
(0)

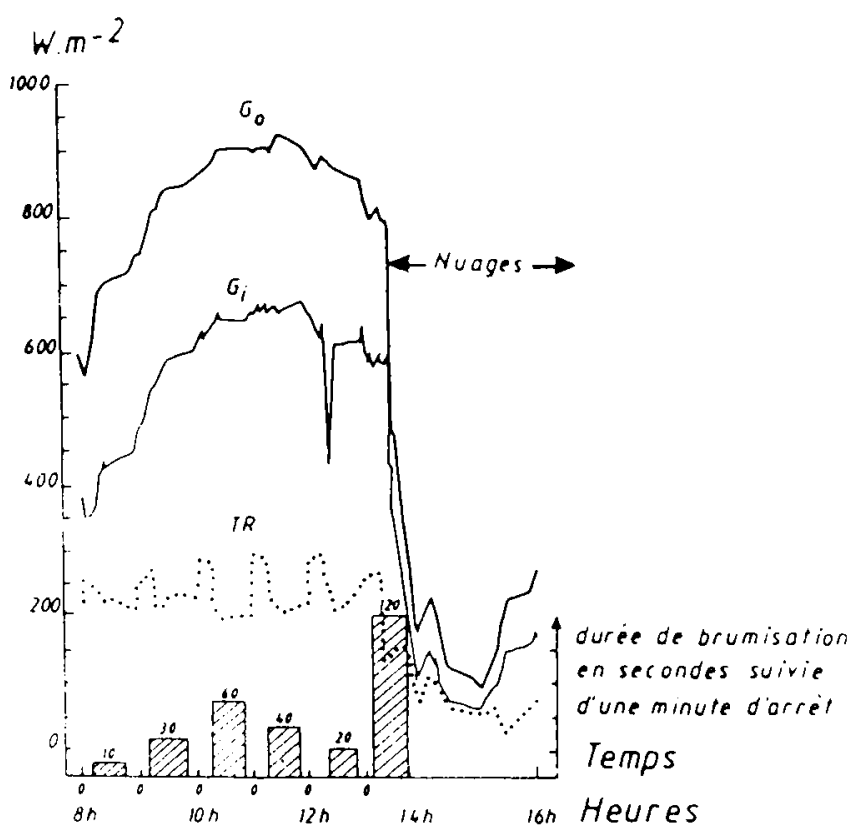

(c)

Température ${ }^{\circ} \mathrm{C}$

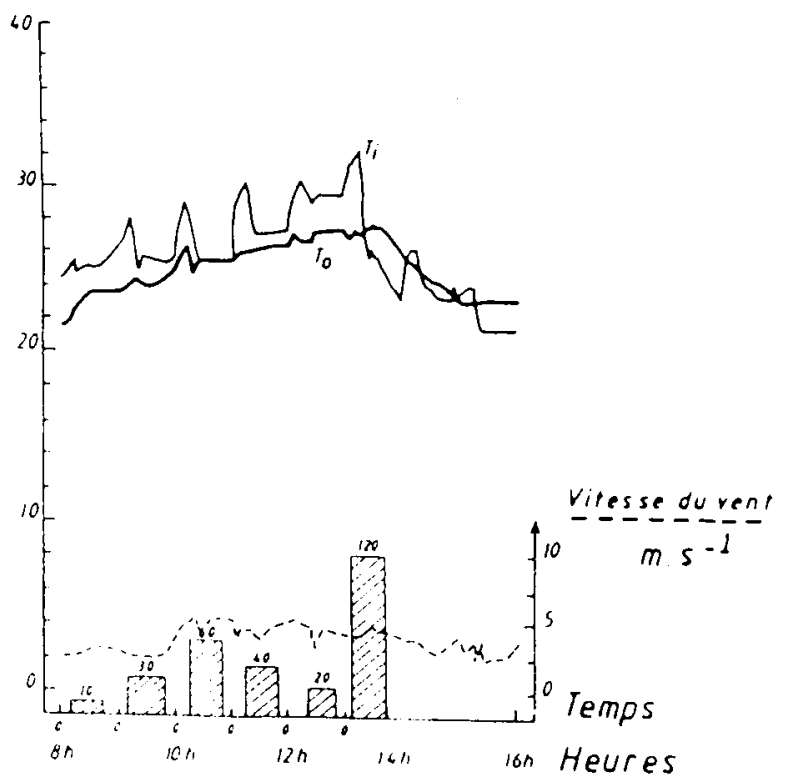

(b)

defisit de saturation

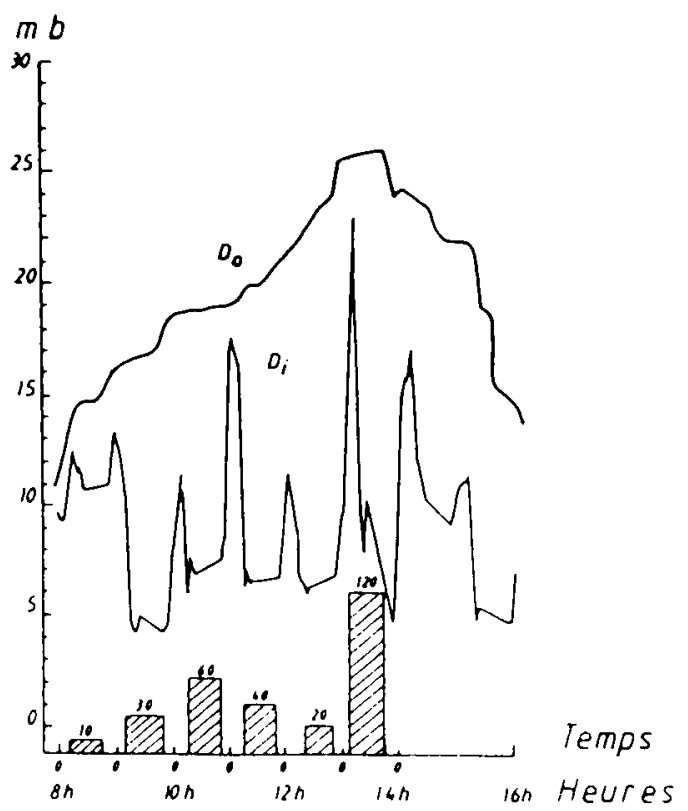

(d)

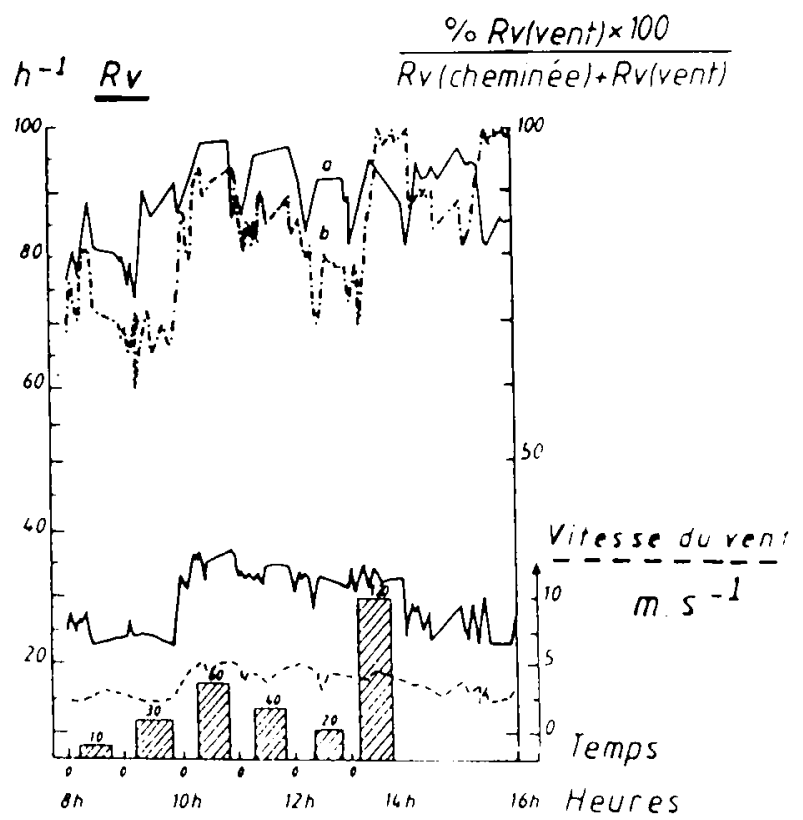

Fig 5. Étude de l'effet de la brumisation. Journée du 31 mai 1989 à Montfavet ( $r=5,3 \%$ ). a) sur la transpiration de la culture $T R$. $G_{0}$ : rayonnement global extérieur, $G_{i}$ : rayonnement global sous serre. b) sur le déficit de saturation de l'air de la serre $D_{i}$. $D_{0}:$ déficit de saturation de l'air extérieur. c) sur la température de serre $T_{i}$. $T_{0}$ : température de l'air extérieur et $V$ vitesse du vent extérieur. d) évolution du renouvellement d'air calculé $R_{v}$ et de sa composante advective. $R_{v}$ (vent)/( $R_{v}$ (cheminée) $+R_{v}$ (vent)), $R_{v}$ (cheminée) étant calculé soit à partir de $\mid D T / / T$ (a) soit à partir de $\left|D T_{v}\right| / T(\mathrm{~b})$.

tion de 2 à 3 mbar seulement) et la diminution de transpiration semble essentiellement due à la diminution du terme radiatif (diminution du rayonnement absorbé par le couvert) et à une augmentation de la résistance stomatique. Les températures de feuille et d'air diminuent légèrement lors de la mise en place de l'écran, l'écart entre ces 2 températures $\left(T_{f}<T_{i}\right)$ restant à peu près constant au cours la journée, que ce soit avec ou sans écran. L'écran d'ombrage ne semble donc pas (du moins, dans les conditions de nos essais, et avec le type d'écran utilisé, qui réfléchit une partie du rayonnement pénétrant dans la serre) inverser la tendance $T_{f}<T_{j}$, alors 

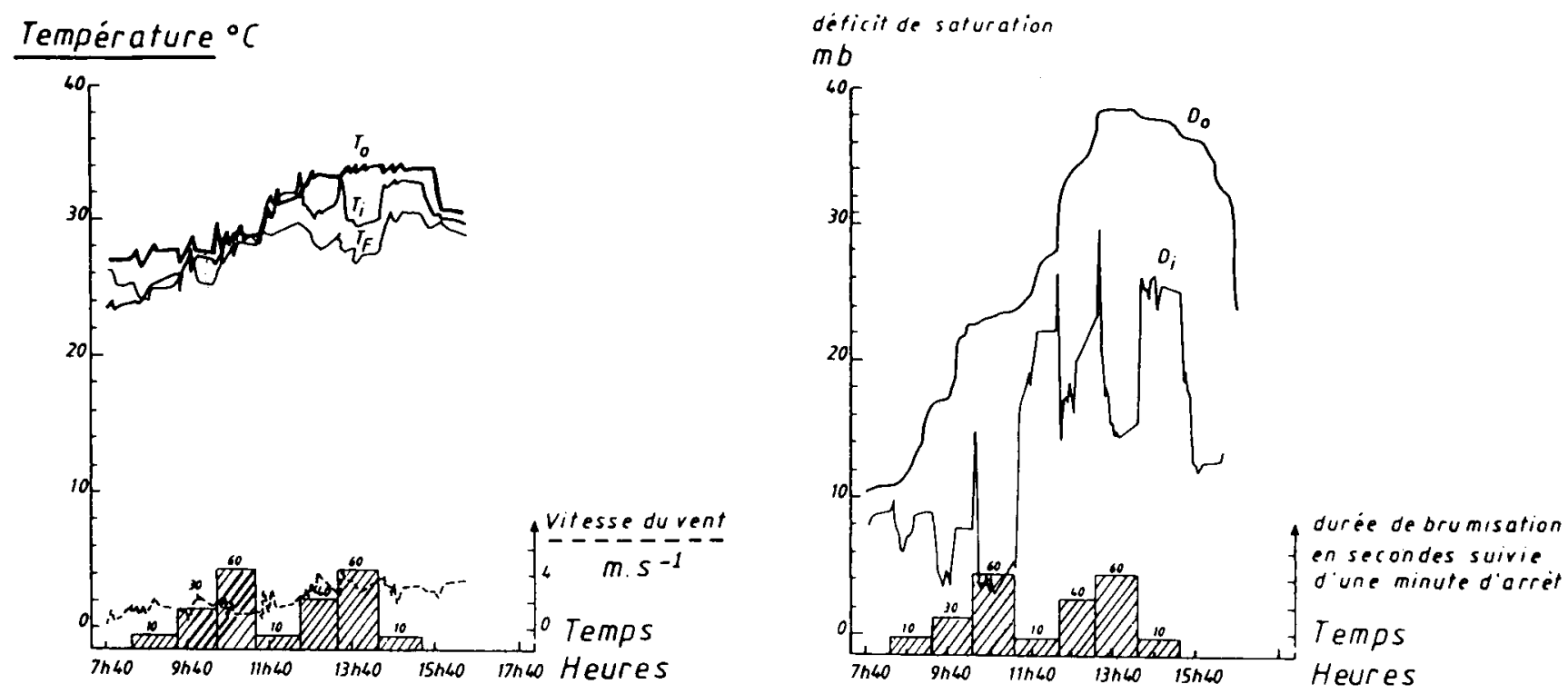

Fig 6. Étude de l'effet de la brumisation Journée du 21 juin 1989 à Montfavet $(r=5,3 \%)$ : a) sur la température d'air de la serre $T_{b}$ et sur la température de feuille $T_{r} T_{0}$ : température de l'air extérieur avec $V$, vitesse du vent extérieur. b) sur $D_{i}$ déficit de saturation de l'air de la serre, $D_{0}$ : déficit de saturation de l'air extérieur.

qu'on a pu observer cette inversion en faisant varier l'angle d'ouverture des ouvrants, dans le cas de l'aération seule.

\section{Aération et brumisation}

Comme l'ombrage, la brumisation réduit significativement le flux de transpiration de la culture, de manière à peu près proportionnelle à l'intensité de brumisation. Son effet porte essentiellement sur la composante advective de TR, puisque le déficit de saturation interne $D_{i}$ subit des baisses très importantes lors des périodes de brumisation. Le rayonnement global incident, $G_{i}$, n'est pratiquement pas modifié (fig $5 \mathrm{c}$ ) par le fin brouillard émis par les buses, qui ne constitue donc pas un "écran radiatif» suffisamment important pour contribuer à une diminution de $T R$. La température d'air dans la serre, $T_{i}$, subit elle aussi des diminutions significatives de l'ordre de $3-4{ }^{\circ} \mathrm{C}$ pour les intensités de brumisation élevées.

Cette diminution de $T_{i}$ se répercute sur $T_{f}$ tant que la diminution du déficit de saturation $D_{i}$ n'est pas très importante. Par contre, la conjonction d'un faible renouvellement d'air (vent faible et/ou faible ouverture des ouvrants) et d'une brumisation intense se traduit par une chute de $D_{i}$ qui freine la transpiration et élève la température des feuilles à une valeur proche de celle de l'air.

L'ensemble de ces résultats montre qu'il serait judicieux, comme l'a suggéré Stanghellini (1987), de gérer le climat de la serre non seulement à partir des variables d'état $\left(T_{i}, D_{i}, T_{o}, D_{o}\right.$, etc), mais aussi en prenant en compte le taux de transpiration de la culture, mesuré (mesure du flux de sève, lysimètre similaire à celui utilisé dans nos essais, etc) ou calculé (modélisation de $T R$ ). On pourrait sans doute tolérer des températures ou des niveaux de rayonnement relativement élevés dans la serre tant que les échanges avec l'extérieur et le taux de transpiration restent eux-mêmes à des niveaux importants. L'écran d'ombrage et la brumisation ne seraient alors utilisés que pendant des situations limites (vent nul, atmosphère extérieure très sèche comme par périodes de mistral ou de tramontane), où la demande climatique risque de devenir supérieure à l'offre de la culture. II reste cependant à définir un critère permettant de définir le moment où il faut déclencher ces équipements auxiliaires, qui diminuent tous 2 la transpiration de manière importante, mais également la photosynthèse dans 
le cas de l'écran d'ombrage. Des mesures de température de surface permettant de calculer un indice de stress par utilisation de l'écart $T_{f}-T_{i}$ (Jackson, 1982) seraient possibles, bien que l'utilisation de radiothermomètres chez un serriste soit difficile à envisager, mais également des capteurs donnant en continu la variation de dimension d'organes (Huguet, 1985; Schoch et al, 1986) pourraient être utilisés pour déterminer le moment adéquat où il faut mettre en œuvre ces équipements auxiliaires.

\section{RÉFÉRENCES}

Baille M, Laury JC, Granger J, Baille A (1985) Étude comparative de deux systèmes d'ombrage. Cah CNIH 1, 10-14

Boulard T (1985) Étude du gisement de chaleur récupérable sur l'air d'une serre, comparaison avec les besoins de chauffage. Note interne INRA, M/85/1. Station de Bioclimatologie d'Avignon
Brun R, Lagier J (1984) Étude d'un nouveau type d'abri mieux adapté au climat méditerranéen. PHM Rev Hortic 235, 29-32

Huguet JG (1985) Appréciation de l'état hydrique d'une plante à partir des variations micrométriques de la dimension des fruits ou des tiges au cours de la journée. Agronomie 5, 733-741

Jackson (1982) Canopy temperature and crop water stress. Advances in Irrigation, vol 1, (D Hillel, ed), Acad Press 43-85

Kabbaj R (1988) Modélisation de la ventilation statique d'une serre. DEA en Energétique de l'Université de Perpignan, $74 p$

Schoch PG, L'hotel JC, Dauple P, Conus G, Fabre MJ (1989) Microvariations de diamètre de tige pour le pilotage de l'irrigation. Agronomie 9, 137-142

Souloumiac D, Itier B (1989) Prise en compte des phénomènes de chaleur latente dans la ventilation naturelle des bâtiments d'élevage. $C R$ Séances Acad Sci Paris, 308, sér II, 269-274

Stanghellini C (1987) Transpiration of greenhouse crops. An aid to climate management. Ph D Dissertation, Agricultural University, Wageningen, 150 $p$ 